\title{
Reproducing Materials of Virtual Elements on Touchscreens using Supplemental Thermal Feedback
}

\author{
Hendrik Richter, Doris Hausen, Sven Osterwald, Andreas Butz \\ $\mathrm{HCl}$ Group \\ University of Munich LMU \\ Amalienstraße 17, 80333 Munich, Germany \\ \{hendrik.richter, doris.hausen, andreas.butz\}@ifi.Imu.de
}

\begin{abstract}
In our everyday life, the perception of thermal cues plays a crucial role for the identification and discrimination of materials. When touching an object, the change of temperature in the skin of our fingertips is characteristic for the touched material and can help to discriminate objects with the same texture or hardness. However, this useful perceptual channel is disregarded for interactive elements on standard touchscreens.

In this paper, we present a study in which we compared the rate of object discrimination for stand-alone thermal stimuli as well as supplemental thermal stimuli characterizing virtual materials on a touchscreen. Our results show that five materials could be discriminated at a stable rate using either stand-alone or supplemental thermal stimuli. They suggest that thermal cues can enable material discrimination on touch surfaces, which gives way for expanded use of thermal stimuli on interactive surfaces.
\end{abstract}

\section{Categories and Subject Descriptors}

H5.2 [User Interfaces]: Haptic I/O

\section{General Terms}

Experimentation

\section{Keywords}

thermal stimuli, material discrimination, remote tactile feedback, interactive surfaces, touch input

\section{INTRODUCTION}

When we rummage in our crammed shoulder bag in order to find the car keys, we hardly use our sense of vision. Instead, we rely on our sense of touch to discriminate objects based on their texture, malleability and temperature. For objects with similar hardness and with a non-textured surface, such as metal and hard-plastics keys, it is primarily

Permission to make digital or hard copies of all or part of this work for personal or classroom use is granted without fee provided that copies are not made or distributed for profit or commercial advantage and that copies bear this notice and the full citation on the first page. To copy otherwise, to republish, to post on servers or to redistribute to lists, requires prior specific permission and/or a fee.

ICMI'12, October 22-26, 2012, Santa Monica, California, USA.

Copyright 2012 ACM 978-1-4503-1467-1/12/10 ...\$15.00.

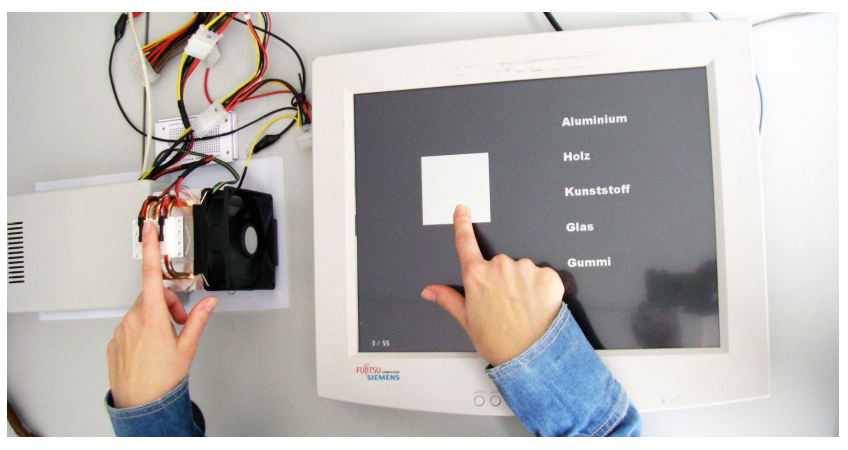

Figure 1: Supplemental thermal cues were given (left hand) to simulate different materials on the touchscreen (right hand).

the characteristic change of temperature in the skin of our fingertips, which lets us discriminate between them.

Thermal stimulation is a rich and emotive perceptual channel, yet underused in human-computer-interaction. This especially holds true for direct touch surfaces. Touching flat and uniformly tempered screens and interactive surfaces with our fingertips or hands has become the de facto standard for interaction with digital interfaces. From mobile devices to tabletops and interactive walls, direct touch is well established because it is cost-efficient, easy to use, and allows for flexible GUI-design. However, the feedback is almost solely based on visual stimuli. Apart from ambiguous vibrotactile buzzes, no information on form, texture, function or state of the virtual input elements is provided haptically. Therefore, researchers have tried to integrate electromechanical actuators into displays to communicate state, form and function of virtual elements. This approach has shown to be greatly beneficial in terms of objective (total task time, accuracy) and subjective (user experience, naturalness) measures of direct touch interaction [3, 11, 15]. The benefits are particularly evident in dynamic multitasking scenarios such as in-vehicle or mobile touch interactions $[18,26]$.

However, our perception of temperature and our ability to identify and discriminate objects solely based on their thermal reaction is still underused on direct touch based interfaces. To this day, temperature feedback for the rendering and simulation of materials is not available on touchscreens. In addition to other tactile stimuli such as vibration or movement, thermal stimuli could help to make buttons and elements on touchscreens more diverse and understand- 
able. Simulated materials such as wood or metal could be part of a touch interface and the additional thermal cues could help to reduce visual and cognitive load.

In this paper, we present the results of a user study in which we use a purpose-built remote tactile interface to simulate thermal characteristics of virtual elements on a touchscreen (see figure 1). Our results show that participants could discriminate five different materials on touch screens solely by their thermal feedback characteristics.

\section{RELATED WORK}

\subsection{Thermal Perception}

In related literature from the fields of physiology, perceptional research or HCI, the term haptic refers to all matters related to the sense of touch. In contrast to the cutaneous sense, which incorporates the skin itself including sensations of pressure, temperature and pain, the common term tactile is mostly used to only describe sensations of pressure [21].

Being classified as a cutaneous sense, perception of thermal cues is produced by either constant (static) or altered (dynamic) skin temperature. For static temperatures between $30-36^{\circ} \mathrm{C}$, no thermal sensation is noted [29]. This range is called physiological zero or neutral zone, because the observer does not experience any thermal stimuli due to adaption [17]. Below $30^{\circ} \mathrm{C}$ and above $36^{\circ} \mathrm{C}$, we perceive a constant sensation of coldness or warmth, respectively. With static temperatures above $45^{\circ} \mathrm{C}$ or below $18^{\circ} \mathrm{C}$, the adaption to thermal cues is not possible and the tactile sensation of temperature is replaced by pain [28]. For dynamic thermal sensations, the perceived stimuli differ depending on the range of temperature. Here, for temperature changes outside the neutral zone, the increasing or decreasing skin temperatures are experienced as increasing or decreasing warmth or coolness [17]. Within the neutral zone, we perceive a rapid increase or decrease of skin temperature as static sensations of warm and cold [30]. Our perception of a material's thermal characteristics is based on these dynamic temperature changes inside the neutral zone.

\subsection{Material Discrimination}

Our skin temperature is ranging from $20^{\circ} \mathrm{C}$ to $40^{\circ} \mathrm{C}$ in daily activity, but typically remains between $32^{\circ} \mathrm{C}$ and $35^{\circ} \mathrm{C}$ [13]. Consequently, the temperature of our skin is usually higher than the temperature of surrounding objects. When we touch a material's surface, heat is transferred away from our skin. The resulting thermal cues are influenced by the material's temperature and the heat flux that is conducted out of the skin. These characteristics depend on thermal conductivity, heat capacity, and initial temperatures of both skin and material [10]. However, studies show that the rates of change have to be over $0.5^{\circ} \mathrm{C} /$ minute to be perceived at all and that there have to be large differences in thermal properties of objects for the user to perceive them [13]. For our perception and discrimination of various materials such as wood or aluminum, the perception of thermal cues is only one of many tactile stimuli providing information. Others include texture, hardness or profile [5].

\subsection{Thermal Feedback in HCI}

In virtual reality or telepresence, thermal feedback has been used in combination with vibration and kinesthetics in order to generate a holistic haptic sensation. Kron et al.[16] combined tactile, kinesthetic, visual and auditory feedback to support a physician's diagnosis on a virtual patient. Caldwell et al.[5] present an instrumented finger, which transmits tactile sensations from a robot to a remote operator. Dionisio [6] constructed an application with multiple forms of thermal feedback using Peltier devices ${ }^{1}$, infrared lamps and ventilators. Thus, thermal characteristics of a virtual scenario are communicated to the user. Oohara et al.[22] suggest to use the whole body as area of application for thermal stimuli. In a recent work, Wilson et al.[30] focused on designing structured thermal feedback on mobile devices to assess how well users perceive thermal notifications on hand and forearm.

Other fields of research deal with the human ability to discriminate objects by the decrease of temperature of the human skin when touching colder materials. Caldwell et al.[5] used a purpose-built instrumented finger to sense and communicate tactile cues from a robot device to the operator. Both Ino et al.[12] and Ho et al.[10] developed stand-alone thermal displays using Peltier elements to simulate the heat flux behavior of touched objects. Their results show that materials with similar heat conductivity, such as copper and stainless steel, were often confused. However, there was no significant difference in the performance of the subjects between identifying real and simulated materials. Ino et al.[12] measured the decrease in finger temperature when in contact with the physical materials aluminum, glass, rubber, polyacrylate and wood. Then, they simulated these cues using their device. Subjects could reliably identify aluminum and wood with rates above $80 \%$, but sometimes confused glass, rubber and polyacrylate. This could result from different individual orderings of the materials from cold to warm. We consider these findings in our evaluation (see chapter 3.2).

\subsection{Tactile Feedback on Touchscreens}

Touch interfaces are ubiquitously used due to their costefficiency, flexibility in GUI-design and ease-of-use. Touchscreens form a de facto standard for the interaction with multi-functional systems such as mobile phones or invehicle-infotainment systems. Still, the feedback that is coming from a touchscreen is mostly visual. The only non-visual feedback that is provided by touchscreens today mostly is a buzz from a vibrating motor or an audible beep. This reduction of multimodal information flowing from the interface to the user results in increased visual and cognitive load [18] and forms a potentially dangerous source of distraction [26].

Therefore, researchers try to utilize the users' sense of touch as an additional information channel. Tactilely conveyed information can be of very diverse nature: e.g. the form, surface structure, malleability, state, meaning, function or distance of a depicted interactive virtual element. Tactile feedback on mobile touchscreen devices has resulted in significantly more text entered per time, fewer errors made and more corrected errors [3, 11]. Other studies show that tactile feedback reduces visual and cognitive load [21, 18]. This especially holds true for dynamic scenarios entailing noise, movement, distraction, attention shifts or high cogni-

\footnotetext{
${ }^{1}$ These thermoelectric heat-pumps consist of two dissimilar conductors, of which one is cooling and the other is warming, depending on the direction and amount of the applied current. The effect of cooling is increased, if the heat is actively transferred away, e.g. by using a cooler fan [7].
} 
tive load [4]. In addition, the provision of palpable stimuli on flat touch surfaces also has beneficial effects on the user's subjective impression $[3,23]$.

However, researchers and designers still struggle with the technical implementation of tactile actuator technology into the touch display [31]. Proposed solutions entail the movement or actuation of the screen as a whole $[14,26,1]$, the segmentation of the interactive surface in individually movable actuator elements (i.e. 'tactile pixels') $[8,24]$ or the use of additional tangible user interfaces atop the touch display [19]. For high resolution tactile feedback, which corresponds to the visual feedback in terms of resolution and scalability, a potentially high number of single actuator elements have to be integrated into the touch device. Most feedback elements only provide one tactile stimulus, which is the same for every touching finger, this hindering from multi-touch tactile feedback. Recent approaches to provide tactile stimuli on arbitrary interactive surfaces are based on separating the area of touch (i.e. fingertip or hand) and resulting tactile stimuli on the user's body. The provision of remote tactile feedback incorporates the use of actuators in the user's direct environment or wearable interfaces. Evaluations indicate positive effects of remote tactile stimuli on user performance and subjective evaluation [20]. The notion of remote tactile feedback can also enable for the simplification of actuator design [25] and the creation of novel tactile modalities by combining different actuators on the user's body [27] . For engineers and designers of touch based interfaces with growing functionality, the incorporation of additional tactile stimuli such as pressure, movement and temperature remains fundamental [31].

However, to our knowledge, thermal cues are still underused on touchscreens as a source of information on the state of the interaction or on virtual elements. Therefore, our goal was to incorporate previous findings on how to simulate materials using defined decreases of temperature [12, 10] and to transfer these findings in order to create discriminable materials on touch-screens. Our approach could help to reduce visual load and to increase user-experience during touch interactions. With the integration of thermal cues as an additional channel of information, the interaction can be greatly enriched.

\section{PROTOTYPE}

The design of the thermal actuator and the characteristics of the five thermal patterns presented (aluminum, glass, rubber, polyacrylate, wood) are based on values obtained in related research projects $[12,10]$. For comparability to related results, we also chose the fingertip as the area of application of the stimuli.

\subsection{Thermal Actuator Device}

The structure of our thermal feedback system is illustrated in figure 2. The actuator is composed of a Peltier element $(29.5 \times 29.5 \times 4.1 \mathrm{~mm})$, which is attached to a $12 \mathrm{~V}$ cooler fan ${ }^{2}$ with heat conducting film. Two analog temperature sensors ${ }^{3}$ are fitted upon the Peltier module next to the touching area to measure the temperature of the heat pump. The Arduino-based ${ }^{4}$ control element reglates the in-

\footnotetext{
${ }^{2}$ RR-910-HTX3-GB

${ }^{3} \mathrm{KTY} 81-210$

${ }^{4}$ www.arduino.cc
}

tensity and direction of the current, which drives the Peltier element. The maximum speed of cooling is around $6.2^{\circ} \mathrm{C} / \mathrm{s}$, heating works with $5.5^{\circ} \mathrm{C} / \mathrm{s}$.

For the evaluation, the thermal stimulus can be given in two ways:

1. The subject rests the index finger of the dominant hand on the Peltier actuator and the temperature decrease is given.

2. The subject touches the virtual interactive element on the touchscreen with the dominant hand, while the associated thermal material information is provided to the non-dominant hand, whose index finger rests on the actuator.
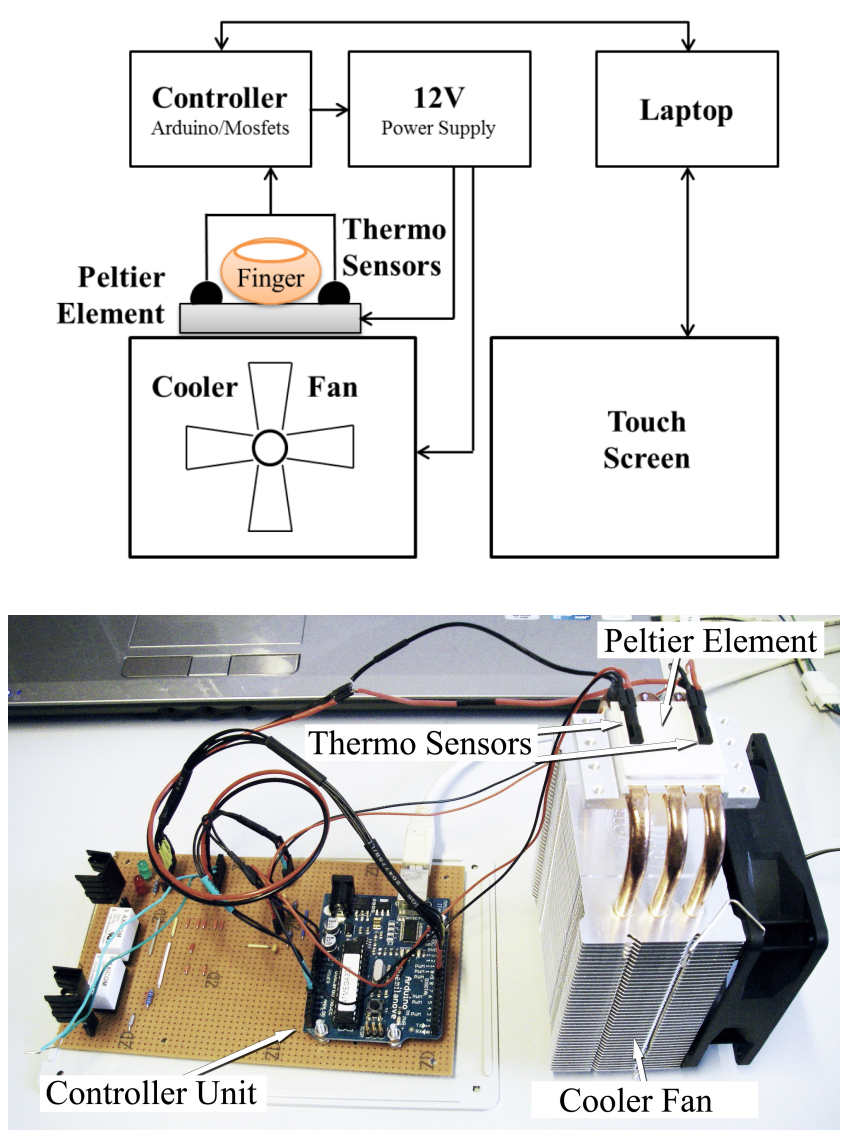

Figure 2: Schematic representation (top) and photo (bottom) of the thermal display prototype.

\subsection{Design of Stimuli}

The measured temperature characteristics of the finger touching the 5 materials are depicted in figure 3. For wood, a small temperature decrease of $0.1^{\circ} \mathrm{C}$ is needed and can be provided by our device in $500 \mathrm{~ms}$. For aluminum, which is the coldest material, a temperature change of $-6.9^{\circ} \mathrm{C}$ is needed [12]. Our device is able to provide this stimulus in $3200 \mathrm{~ms}$ when cooling down from the starting temperature of $30^{\circ} \mathrm{C}$. For each material, the cooling process was preprogrammed. 


\begin{tabular}{lcc}
\hline material & $\Delta \mathrm{T}$ & cooling time \\
\hline wood & $-0.1^{\circ} \mathrm{C}$ & $500 \mathrm{~ms}$ \\
polyacrylate & $-1.7^{\circ} \mathrm{C}$ & $600 \mathrm{~ms}$ \\
rubber & $-2.6^{\circ} \mathrm{C}$ & $1000 \mathrm{~ms}$ \\
glass & $-3.1^{\circ} \mathrm{C}$ & $1200 \mathrm{~ms}$ \\
aluminum & $-6.9^{\circ} \mathrm{C}$ & $3200 \mathrm{~ms}$ \\
\hline
\end{tabular}

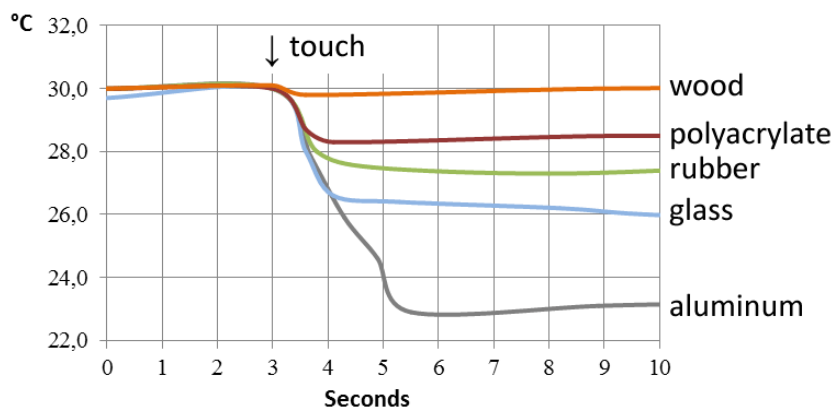

Figure 3: Measured temperature decreases $(\Delta T)$ and corresponding cooling times for the five applied temperatures (top). Resulting temperature decreases for the five materials (bottom).

\section{EVALUATION}

The objective of our evaluation was twofold: First, we wanted to assay whether participants were able to discriminate virtual materials using our stand-alone prototype. Second, we were interested in evaluating the participants' ability to discriminate virtual objects on touchscreens using the prototype as a supplemental tactile feedback device. In both parts of this experiment we evaluated the identification rate for five virtual materials based on predefined thermal cooldown characteristics.

\subsection{Measuring Finger Temperature}

According to related work, the temperature of the finger remains between $25^{\circ} \mathrm{C}$ to $36^{\circ} \mathrm{C}[13,10]$ and is highly individual. In a preliminary evaluation, we asked 18 participants (13 male) to hold an analog temperature sensor ${ }^{5}$ between the thumb and index finger of their dominant hand for 30 seconds (see figure 4). The results show a mean of $30.01^{\circ} \mathrm{C}$ $(\mathrm{SD}=2.79)$ and a median of $30.62^{\circ} \mathrm{C}$. Consequently, we used $30.0^{\circ} \mathrm{C}$ as the resting temperature of the fingertip during the following evaluations.

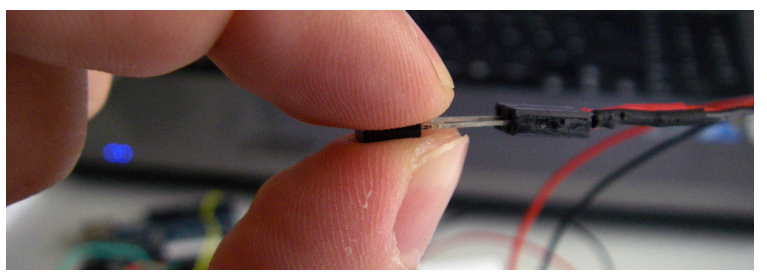

Figure 4: In a prestudy, the skin's temperature of the fingertip was measured.

${ }^{5} \mathrm{KTY} 81-210$

\subsection{Research Questions and Hypotheses}

As stated before, the temperature decreases measured from real materials [12] served as a basis for our evaluation. The thermal feedback device and the thermal stimuli were implemented accordingly. Our main goal is to use the applied thermal stimuli to augment interactive touchscreenelements with additional tactile information on their materiality. Hence, we formulated the following two hypotheses:

H1: Material identification by thermal cues is possible and scores are not significantly different for stand-alone and supplemental thermal stimuli (feedback on the nondominant hand resulting from a touch interaction with the dominant hand).

H2: Subjective ratings of realism, signal-design and information-transfer of the thermal stimuli are comparable for stand-alone and supplemental thermal stimuli.

\subsection{Experimental Design}

We used a within-subject/repeated-measures design. The independent variables were feedback location (index finger's tip of dominant hand vs. non-dominant hand) and the applied stimulus (five defined temperature changes $(\Delta T)$ for aluminum, glass, rubber, polyacrylate and wood). As the dependent variable we recorded the user's decision for a material.

\subsection{Apparatus}

As shown in figure 5, we used a thermal feedback device and a touch screen (capacitive single touch) for the study. The set-up was mirrored for left-handed persons. A list of the five materials was presented on the screen and was randomized for every trial.
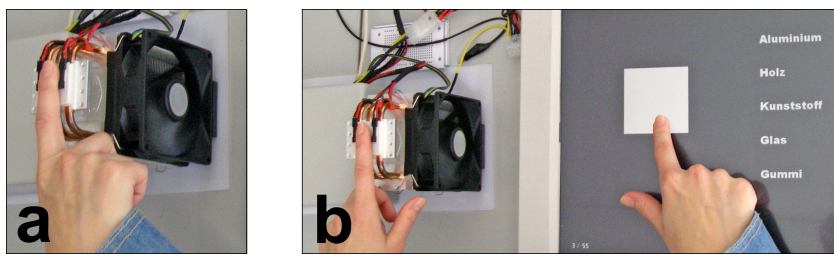

Figure 5: The two uses of the thermal display during the evaluation: (a) the stand-alone thermal display is used to present cues to the dominant hand; (b) the dominant hand interacting on the screen with the supplemental thermal display presenting cues to the non-dominant hand.

For stand-alone thermal feedback, the seated participant's index finger of the dominant hand was rested on the thermal display. Upon touch, the subject was given a thermal cue. Subsequently, the perceived stimulus had to be selected by touching the list on the touchscreen. For supplemental applied stimuli, the participant's index finger of the non-dominant hand was rested on the device and stimuli were given when the user touched the white rectangle on the screen with his dominant hand. When the participants had decided on a material, they selected it from the list. For both parts, the system measured the duration of stimulus presentation and registered the user's selection. Every material was given eleven times in a randomized sequence for every subject. In order to calibrate participants to the task, the first five selections contained each of the five materials. These selections were not measured. The order in 
which the two tests had to be performed was counterbalanced across subjects. Additionally, we analyzed the participants' subjective evaluation of both types of stimuli using a questionnaire. We measured the evaluation of the signal's realism, signal design and information bandwidth by adapting AttrakDiff, an evaluation method stemming from user experience research [9]. The method is originally used for measuring the user's opinion on pragmatic quality, hedonic quality and attractiveness of a product. In our experiment, participants rated the stimuli on a 7 -point scale of semantic differentials, i.e. pairs of opposing adjectives.

\subsection{Participants}

A total number of 20 subjects (nine female) participated in the user study. They were aged from 20 to 29 (average age 24). Two subjects declared to be left-handed.

\subsection{Procedure}

At the beginning, the users were asked to fill out a demographic questionnaire on age, sex, handedness, diseases or finger-injury, which could affect the measurement. Next, participants were informed about the five available virtual materials (Aluminum, Glass, Rubber, Polyacrylate and Wood) and were asked to rank them from cold to warm according to their personal experience. For the stand-alone thermal feedback task, the defined temperature decrease was started as soon as the subject touched the Peltier element directly. Participants could take as much time for the decision as they wanted, but were only allowed to touch the element once for each decision. Once the subjects had decided on the material, they lifted their finger from the device and selected the perceived material from the list on the touch-screen. The experimenter made sure that there was a two-second break in order for the element to reheat to the resting temperature of $30^{\circ} \mathrm{C}$. For the supplemental thermal feedback task on the touchscreen, the participants interacted on the screen with their dominant hand. Participants were asked to touch the virtual white rectangle on the screen. A synchronized thermal feedback (temperature decrease) was given and the reduced temperature was held as long as the participant touched the material. When they lifted their finger from the square on the touchscreen, the device's temperature was heated back to $30^{\circ} \mathrm{C}$. The perceived material had to be selected from the list on the touch-screen. After both tasks, participants were asked to fill out the semantic differential questionnaires about the signal's realism, signal design and information bandwidth. Finally, the participants were asked in a semi-structured interview about other possible locations and uses of thermal feedback.

\section{RESULTS}

\subsection{Material Identification}

Figure 6 shows the rates of material discrimination for both stand-alone thermal stimuli and supplemental thermal feedback. For stand-alone thermal feedback, identification rates differ between individual materials. Aluminum (identification rate: $65.5 \%$ ) was most reliably identified followed by glass $(39.0 \%)$, polyacrylate $(36.5 \%)$ and wood $(33.0 \%)$, which are nearly equally well identified. Rubber was identified in only $14 \%$ of all cases. In other words, identification of rubber was worse than the chance level of $20 \%$ when selecting one material out of five, while all other materials were

\begin{tabular}{|c|c|c|c|c|c|}
\hline & $\begin{array}{l}\Sigma \\
\text { ○ } \\
\text { ○ }\end{array}$ & 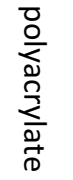 & $\begin{array}{l}\bar{T} \\
\frac{\mathrm{v}}{0} \\
\frac{\mathrm{D}}{\mathrm{D}}\end{array}$ & $\frac{\sigma a}{\tilde{N}}$ & $\begin{array}{l}\frac{0}{\equiv} \\
\frac{2}{3} \\
\frac{5}{5} \\
\frac{5}{3}\end{array}$ \\
\hline & \multicolumn{5}{|c|}{ stand-alone feedback } \\
\hline wood & 33.0 & 28.5 & 37.0 & 1.0 & 0.5 \\
\hline polyacrylate & 31.0 & 36.5 & 24.0 & 6.0 & 2.5 \\
\hline rubber & 21.0 & 36.5 & 14.0 & 21.0 & 7.5 \\
\hline glass & 16.0 & 23.0 & 11.0 & 39.0 & 11.0 \\
\hline \multirow[t]{2}{*}{ aluminum } & 0.5 & 0.5 & 1.5 & 32.0 & 65.5 \\
\hline & \multicolumn{5}{|c|}{ supplemental feedback } \\
\hline wood & 43.5 & 30.0 & 22.5 & 3.0 & 1.0 \\
\hline polyacrylate & 18.5 & 45.5 & 17.5 & 14.0 & 4.5 \\
\hline rubber & 12.5 & 36.5 & 10.0 & 33.0 & 8.0 \\
\hline glass & 10.5 & 21.0 & 9.0 & 45.5 & 14.0 \\
\hline aluminum & 0.5 & 2.5 & 5.0 & 21.5 & 70.5 \\
\hline
\end{tabular}

Figure 6: Identification scores ("percentage of correct identifications") for both stand-alone and supplemental thermal stimuli.

identified clearly above chance level. Looking at the results for supplemental feedback on the touch screen, aluminum is identified best $(70.5 \%)$, followed by glass $(44.5 \%)$, polyacrylate $(45.5 \%)$ and wood $(43.5 \%)$. Once again rubber is identified below chance level with $10 \%$. Although identification means are a bit higher for all cases (except rubber), there is no statistical significant difference $(\mathrm{p}=0.060)$ between stand-alone and supplemental feedback.

Thus, we can confirm H1.

Our results are comparable to those of Ino et al.[12] (see figure 7 ). However, they used a small sample of five participants whereas we incorporated 20 subjects. Our observations concerning the order of identification (aluminum was best identified, while rubber was hardly identified) is also visible in Ino's results.

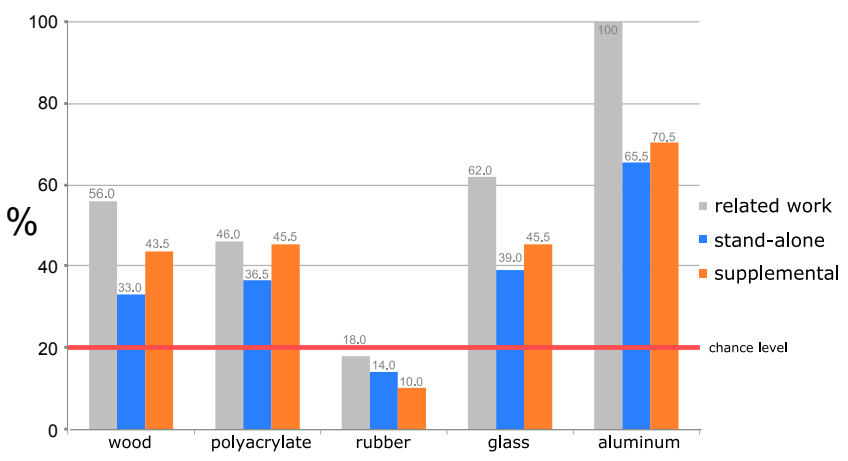

Figure 7: Identification scores in percent by related work (Ino et al.[12]), and using our standalone and supplemental setup. 


\begin{tabular}{|r|c|c|c|}
\hline & \multicolumn{3}{|c|}{ stand-alone feedback } \\
\hline order & used & investigated & individual \\
\hline wood & 33.0 & 37.0 & 44.5 \\
\hline polyacrylate & 36.5 & 31.0 & 40.5 \\
\hline rubber & 14.0 & 36.5 & 48.0 \\
\hline glass & 39.0 & 39.0 & 45.0 \\
\hline aluminum & 65.5 & 65.5 & 78.0 \\
\hline average & $\mathbf{3 7 . 6}$ & $\mathbf{4 1 . 8}$ & $\mathbf{5 1 . 2}$ \\
\hline & \multicolumn{3}{|}{} \\
\hline & \multicolumn{3}{|c|}{ supplemental feedback } \\
\hline order & used & investigated & individual \\
\hline wood & 43.5 & 22.5 & 32.0 \\
\hline polyacrylate & 45.5 & 18.5 & 33.5 \\
\hline rubber & 10.0 & 36.5 & 42.5 \\
\hline glass & 45.5 & 45.5 & 54.5 \\
\hline aluminum & 70.5 & 70.5 & 78.5 \\
\hline average & $\mathbf{4 3 . 0}$ & $\mathbf{3 8 . 7}$ & $\mathbf{4 8 . 2}$ \\
\hline
\end{tabular}

Figure 8: Comparison of identification scores for the material order used in the evaluation, for the investigated average order and for individual order for each participant.

In summary, we can state that we achieved stable rates of discrimination for either stand-alone or supplemental thermal feedback. Results show no significant differences between the results for directly applied stimuli and stimuli that were given to the non-interacting hand. In tendency, participants were even better in discriminating materials on touchscreens than with the stand-alone device, even though the touchscreen did not show additional information about the material.

\subsection{Effect of ordering}

For our analysis, we used the order of materials proposed by Ino et al.[12] (from cold to warm: aluminum, glass, rubber, polyacrylate, wood). Having asked our participants about their personal order of the materials, however, it became obvious that most users expected a different ordering. Looking at all different orders we can observe two groups of materials, generally cold materials (aluminum and glass) and generally warm materials (wood, polyacrylate and rubber). While participants' answers differ in the order of materials in one material group, no participant ordered the materials in a sequence mixing cold and warm materials. Figure 8 shows the identification rates based on the order of Ino et al.[12] we used in our evaluation (depicted by two of our participants), investigated order (averaged order based on all participants from cold to warm: aluminum, glass, polyacrylate, wood, rubber; depicted by five of our participants) and individual order for every participant. With the exception of supplemental feedback in investigated order, all identification rates increase. Our observations show the clearly individual nature of thermal material perception.

\subsection{Subjective ratings}

Subjects were asked to rate realism, signal design and information bandwidth of both stand-alone and supplemental

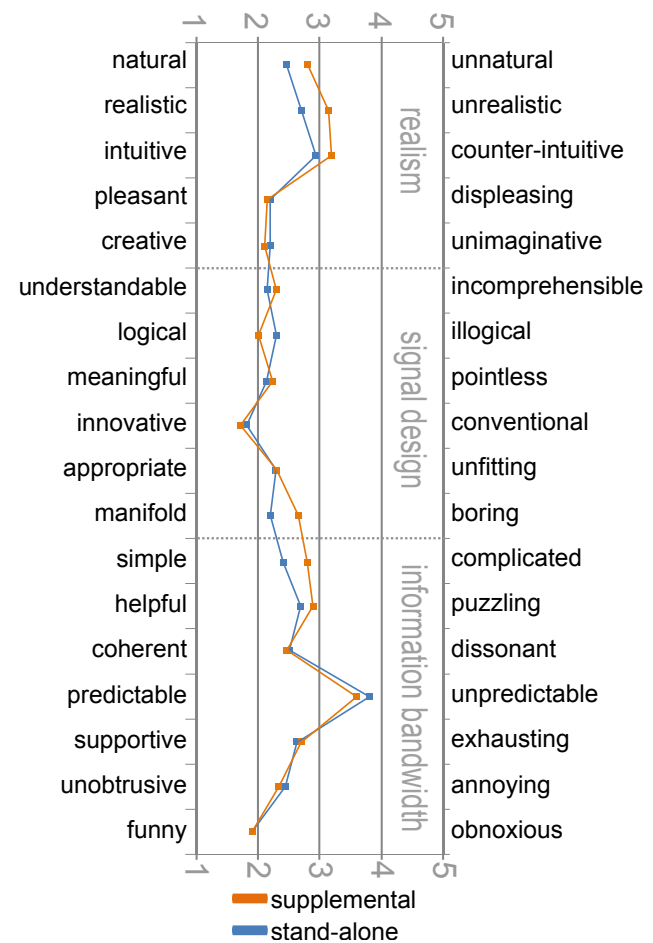

Figure 9: Average results of subjective ratings of stand-alone and supplemental thermal stimuli, positive adjectives left. Discrete values are connected for readability.

thermal stimuli on a five-grade semantic differential. The results are depicted in figure 9. Both stand-alone and supplemental stimuli were perceived as being pleasant and creative (realism); understandable, logical, meaningful and innovative (signal-design) and even fun to use (information). Ratings below 3 for thermal cues were given for being far from reality and difficult to understand (realism). Subjects also described the stimuli as being unpredictable (information). In general, the subjective ratings for both stand-alone and supplemental thermal stimuli showed no difference of more than one grade. Small differences were stated for supplemental stimuli as being less natural (realism), not manifold (signal design) and complicated in comparison to direct stimuli (information). In summary, both types of signal communication obtained similar values, thus affirming H2.

\section{FUTURE WORK}

We used off-the-shelf materials to implement our actuator system. Consequently, our device has certain limitations, such as the relatively large amount of energy that is needed to generate the thermal stimuli. The size of the actuator currently impedes the implementation on mobile devices. We assume that in future implementations the actuators will be smaller and the cool-down and reheating process will be faster due to improved actuator technology (e.g. higher amperage, enhanced thermal conduction). This could result in improved discrimination rates and would allow for an even greater range of discriminable materials. We will repeat our evaluation with more participants in order to gain more significant and stable results. 


\section{DISCUSSION}

We used a supplemental actuator device to simplify the implementation of thermal cues on touch surfaces; no dedicated thermal display for every single pixel of the interactive surface is needed. Previous work has shown the potential of remote tactile interfaces to create novel haptic stimuli by combining different actuators (e.g. vibrotactile, mechanical, thermal) on the user's body [27].

It was interesting to see that the notion of remote tactile feedback did not have a negative effect on discrimination rates. Discrimination rates were even better for 4 out of 5 materials. The perception of a reference value is a possible explanation for this: When supplemental thermal feedback is given, our finger remained on the prototype, so we perceive both the active cooling and the active heating of the element. The temperature of the finger on the touchscreen did not change, thus a constant reference value was given. The nature of our receptors may have helped to detect the slight temperature changes between the index fingers of both hands [13].

We also discovered a strong effect of the personal ordering of materials in temperatures from cold to warm. When taking the users' personal estimation of object temperatures into account, the rates for detecting materials increased considerably. Some people consider aluminum to be colder than glass, while others thought it was the other way around. This documents the clearly individual nature of tactile perception and should be considered in future implementations on personal touch displays such as on mobile devices or incar touchscreens. This could help to generate a more life-like impression of the virtual materials.

\subsection{Applications}

When spatially separating touch input and resulting tactile feedback, we have to incorporate the psychophysics of temperature perception and varying density of thermal receptors in the human skin. Thermal feedback can be given using wearable actuators or by instrumenting the user's environment. Better than mechanical stimuli such as movement or vibration, thermal cues can also be provided 'over a distance', e.g. using warm air jets. We can think of using the forearm, the back or thighs for thermal cues during touch interactions; e.g. by instrumenting the frame of an interactive table, the steering wheel or the driver's seat in the car.

\section{Material Simulation.}

First of all, supplemental thermal cues could be used to support the non-visual exploration of an interactive surface. Thermal cues could help to make GUI objects discriminable, thus helping to reduce visual and cognitive load. This could be especially helpful in dynamic scenarios such as mobile device interaction or the driver's interaction with touch based in-vehicle infotainment systems. Here, thermal stimuli should be used in addition to other tactile information (e.g. vibration, movement) representing material and surface characteristics to establish a rich and redundant representation of virtual elements.

\section{Additional Information.}

Furthermore, thermal cues could also communicate semantic information such as state, function or proximity of a virtual element. Virtual touchscreen buttons could feel hot or cold to inform the user about their important function ("Buy ticket?"). Icons of data files or applications could feel cold when not used for a long time. Being a personal and private channel of information, supplemental thermal cues are appropriate for collaborative and multi-user scenarios involving tabletops or interactive walls. The temperature of data objects could communicate its ownership ("It's hot so it's not mine!"). It is important to consider that with supplemental thermal stimuli (and user identification), an object can have different temperatures for different users. The feedback is interlinked with the user rather than the touch surface.

\section{Thermons.}

Finally, thermal cues could also be used without touch interfaces. They could serve as structured, abstract messages communicating information non-visually. Brewster et al. have established the concept of 'Tactons' or tactile icons to support users of desktop interfaces and mobile devices as well as visually impaired users with a variety of tactile information [2]. For thermal cues, a similar principle could be developed. Useful parameters are absolute temperature, rate of temperature change or total temperature difference (see chapter 2.1). We propose the term 'Thermons' for structured thermal messages or 'thermal icons'.

\section{CONCLUSIONS}

In summary, our results show that virtual touchscreen elements are discriminable based on thermal feedback. To a certain extent, even a simulation of materials is possible. We exemplified an easy and versatile implementation of a supplemental thermal interface. Our results will enable the future exploitation of our perception of temperature to naturalize and enrich the interaction with touch surfaces.

\section{REFERENCES}

[1] O. Bau, I. Poupyrev, A. Israr, and C. Harrison. TeslaTouch. In Proceedings of the 23nd annual ACM symposium on User interface software and technology - UIST '10, page 283. ACM Press, 2010.

[2] S. Brewster and L. M. Brown. Tactons: structured tactile messages for non-visual information display. In Proceedings of the fifth conference on Australasian user interface - AUIC '04, pages 15-23, 2004.

[3] S. Brewster, F. Chohan, and L. Brown. Tactile feedback for mobile interactions. In Proceedings of the SIGCHI conference on Human factors in computing systems - CHI '07, page 159. ACM Press, 2007.

[4] J. L. Burke, M. S. Prewett, A. A. Gray, L. Yang, F. R. B. Stilson, M. D. Coovert, L. R. Elliot, and E. Redden. Comparing the effects of visual-auditory and visual-tactile feedback on user performance. Proceedings of the 8th international conference on Multimodal interfaces - ICMI '06, page 108, 2006.

[5] D. Caldwell. Enhanced tactile feedback (tele-taction) using a multi-functional sensory system. Robotics and Automation, pages 955-960, 1993.

[6] J. Dionisio. Virtual hell: A trip through the flames. IEEE Computer Graphics and Applications, 17:11-14, 1997.

[7] S. Godfrey. An introduction to thermoelectric coolers. http://www.electronics-cooling.com/1996/09/ 
an-introduction-to-thermoelectric-coolers/, Sept. 1996.

[8] C. Harrison and S. Hudson. Providing dynamically changeable physical buttons on a visual display. In Proceedings of the 27 th international conference on Human factors in computing systems - CHI'2009, pages 299-308. ACM, 2009.

[9] M. Hassenzahl, M. Burmester, and F. Koller. AttrakDiff: ein Fragebogen zur Messung wahrgenommener hedonischer und pragmatischer Qualität. In Proceedings of Mensch \& Computer 2003: Interaktion in Bewegung, pages 187-196. B. G. Teubner, 2003.

[10] H. Ho and L. Jones. Material identification using real and simulated thermal cues. Annual International Conference of the IEEE Engineering in Medicine and Biology Society., 4:2462-5, 2004.

[11] E. Hoggan, S. A. Brewster, and J. Johnston. Investigating the effectiveness of tactile feedback for mobile touchscreens. In Proceeding of the twenty-sixth annual CHI conference on Human factors in computing systems - CHI '08, page 1573, 2008.

[12] S. Ino, S. Shimizu, T. Odagawa, M. Sato, M. Takahashi, T. Izumi, and T. Ifukube. A tactile display for presenting quality of materials by changing the temperature of skin surface. In Robot and Human Communication, 1993. Proceedings., 2nd IEEE International Workshop on, pages 220 -224, 1993.

[13] L. A. Jones and M. Berris. The psychophysics of temperature perception and thermal-interface design. In Proceedings of the 10th Symposium on Haptic Interfaces for Virtual Environment and Teleoperator Systems, HAPTICS '02, page 137. IEEE Computer Society, 2002.

[14] T. Kaaresoja and L. Brown. Snap-Crackle-Pop: Tactile feedback for mobile touch screens. volume 6 , pages 565-566, 2006.

[15] E. Koskinen, T. Kaaresoja, and P. Laitinen. Feel-good touch: finding the most pleasant tactile feedback for a mobile touch screen button. In Proceedings of the 10th international conference on Multimodal interfaces, ICMI '08, pages 297-304. ACM, 2008.

[16] A. Kron and G. Schmidt. Multi-Fingered Tactile Feedback from Virtual and Remote Environments. In Proceedings of the 11th Symposium on Haptic Interfaces for Virtual Environment and Teleoperator Systems (HAPTICS'03), HAPTICS '03, pages 16-. IEEE Computer Society, 2003.

[17] S. Lederman and R. Klatzky. The hand as a perceptual system. Clinics in Developmental Medicine, pages 16-35, 1998.

[18] R. Leung, K. MacLean, M. B. Bertelsen, and M. Saubhasik. Evaluation of haptically augmented touchscreen gui elements under cognitive load. In Proceedings of the ninth international conference on Multimodal interfaces - ICMI '07, volume 07, page 374. ACM Press, 2007.

[19] N. Marquardt, M. A. Nacenta, J. E. Young, S. Carpendale, S. Greenberg, and E. Sharlin. The haptic tabletop puck: tactile feedback for interactive tabletops. In Proceedings of the ACM International
Conference on Interactive Tabletops and Surfaces, ITS '09, pages 85-92. ACM, 2009.

[20] C. McAdam and S. Brewster. Distal tactile feedback for text entry on tabletop computers. In Proceedings of the 2009 British Computer Society Conference on Human-Computer Interaction, pages 504-511. British Computer Society, 2009.

[21] I. Oakley, M. R. McGee, S. Brewster, and P. Gray. Putting the feel in 'look and feel'. In Proceedings of the SIGCHI conference on Human factors in computing systems, CHI '00, pages 415-422. ACM, 2000.

[22] J. Oohara, H. Kato, Y. Hashimoto, and H. Kajimoto. Presentation of positional information by heat phantom sensation. In Proceedings of the 2010 international conference on Haptics - generating and perceiving tangible sensations: Part II,

EuroHaptics'10, pages 445-450. Springer-Verlag, 2010.

[23] I. Poupyrev, S. Maruyama, and J. Rekimoto. Ambient touch: designing tactile interfaces for handheld devices. In Proceedings of the 15th annual ACM symposium on User interface software and technology, UIST '02, pages 51-60. ACM, 2002.

[24] I. Poupyrev, T. Nashida, S. Maruyama, J. Rekimoto, and Y. Yamaji. Lumen: interactive visual and shape display for calm computing. In ACM SIGGRAPH 2004 Emerging technologies, SIGGRAPH '04, page 17. ACM, 2004.

[25] H. Richter, B. Blaha, A. Wiethoff, D. Baur, and A. Butz. Tactile feedback without a big fuss: simple actuators for high-resolution phantom sensations. In Proceedings of the 13th international conference on Ubiquitous computing, UbiComp '11, pages 85-88. ACM, 2011.

[26] H. Richter, R. Ecker, C. Deisler, and A. Butz. Haptouch and the $2+1$ state model: potentials of haptic feedback on touch based in-vehicle information systems. In Proceedings of the 2nd International Conference on Automotive User Interfaces and Interactive Vehicular Applications, AutomotiveUI '10, pages 72-79. ACM, 2010.

[27] H. Richter, S. Löhmann, and A. Wiethoff. Hapticarmrest: remote tactile feedback on touch surfaces using combined actuators. In Proceedings of the Second international conference on Ambient Intelligence, AmI'11, pages 1-10. Springer-Verlag, 2011.

[28] R. J. Schepers and M. Ringkamp. Thermoreceptors and thermosensitive afferents. Neuroscience and Biobehavioral Reviews, pages 177-184, 2010.

[29] R. Verrillo, S. Bolanowski, C. Checkosky, and F. McGlone. Effects of hydration on tactile sensation. Somatosensory and Motor Research, pages 93-108, 1998.

[30] G. Wilson, M. Halvey, S. A. Brewster, and S. A. Hughes. Some like it hot: thermal feedback for mobile devices. In Proceedings of the 2011 annual conference on Human factors in computing systems, CHI '11, pages 2555-2564. ACM, 2011.

[31] A. Wright. The touchy subject of haptics. Commun. ACM, 54(1):20-22, Jan. 2011. 